\title{
CLASS NUMBER 5, 6 AND 7
}

\author{
CHRISTIAN WAGNER
}

ABStRaCt. We outline the determination of all imaginary quadratic fields with class number 5,6 or 7 .

\section{INTRODUCTION ${ }^{1}$}

In this paper we outline the proof that the following table contains all discriminants $-d<0$ of imaginary quadratic fields with class number $h=5,6$ or 7 .

TABLE 1. Fundamental discriminants with class number 5,6 or 7

\begin{tabular}{||l|l||}
\hline$h$ & \multicolumn{1}{|c||}{$d,-d$ (fundamental) discriminant, $h(-d)=h$} \\
\hline \hline 5 & $47,79,103,127,131,179,227,347,443,523,571,619,683,691,739,787$, \\
& $947,1051,1123,1723,1747,1867,2203,2347,2683$ \\
\hline 6 & $87,104,116,152,212,244,247,339,411,424,436,451,472,515,628,707$, \\
& $771,808,835,843,856,1048,1059,1099,1108,1147,1192,1203,1219,1267$, \\
& $1315,1347,1363,1432,1563,1588,1603,1843,1915,1963,2227,2283,2443$, \\
& $2515,2563,2787,2923,3235,3427,3523,3763$ \\
\hline 7 & $71,151,223,251,463,467,487,587,811,827,859,1163,1171,1483,1523$, \\
& $1627,1787,1987,2011,2083,2179,2251,2467,2707,3019,3067,3187$, \\
& $3907,4603,5107,5923$ \\
\hline
\end{tabular}

Recently, S. Arno [1, 2] combined methods of H. Stark [16] for $h=2$ and H. Montgomery and P. Weinberger [11] for $h=2$ and 3 to solve the class number-4 problem ${ }^{2}$.

Arno had to overcome the problem of $d$ having up to three distinct prime divisors, but could profit from the small number of leading coefficients of the reduced binary quadatic forms. In our case, $d$ is simplified to at most two prime divisors, but the larger number of coefficients results in certain estimates being much worse. Therefore, we have to combine the abovementioned methods in another way as Arno. For $h=8$ both items take a negative turn: up to four prime divisors and eight coefficients, which makes this case seem impregnable.

Received by the editor December 16, 1993 and, in revised form, February 13, 1995.

1991 Mathematics Subject Classification. Primary 11R29.

Key words and phrases. Binary quadratic form, class number, discriminant, imaginary quadratic field.

${ }^{1}$ General information on Gauss' class number problem may be obtained from the articles by S. Böcherer [3], D. Goldfeld [6], J. Oesterlé [13] and J.-P. Serre [14].

${ }^{2}$ As the referee pointed out to me, S. Arno has now extended some of these results. Arno has treated the class number problem for all odd $h$ from 5 to 23 . 


\section{Class Number, DisCRIminant AND FORMS}

The restriction of $h$ to the values 5,6 or 7 implies conditions that must be fulfilled by the discriminants and the leading coefficients in their reduced (binary quadratic) forms, which occur in certain formulas of the abovementioned methods. In this section we list some of these conditions. This will help us later in reducing the computer work for searching for discriminants with class number 5,6 or 7 .

We are interested in reduced forms $f(x, y)=a x^{2}+b x y+c y^{2}$ with discriminant $-d$, i.e., forms with $-d=b^{2}-4 a c$ and $-a<b \leq a<c$ or $0 \leq b \leq a=c$ (which implies $a \leq \sqrt{d / 3})$. An example is the principal form

$$
f_{1}(x, y)= \begin{cases}x^{2}+\frac{d}{4} y^{2} & \text { for } d \equiv 0 \quad(\bmod 4) \\ x^{2}+x y+\frac{d+1}{4} y^{2} & \text { for } d \equiv 3 \quad(\bmod 4)\end{cases}
$$

which is the only reduced form with 1 as leading coefficient $a$.

Throughout this paper we let $f_{i}(x, y)=a_{i} x^{2}+b_{i} x y+c_{i} y^{2}, i=1, \ldots, h$, be the reduced forms with discriminant $-d$, numbered in a way that their leading coefficients $1, a_{2}, a_{3}, \ldots, a_{h}$ are in ascending order, i.e., $1<a_{2} \leq a_{3} \leq \cdots \leq a_{h}$.

Lemma 2.1. Let $f(x, y)=a x^{2}+b x y+c y^{2}$ be a reduced form with discriminant $-d$. Then $y \neq 0$ implies $f(x, y) \geq c$, and $y=0, x \neq 0$ implies $f(x, y) \geq a$.

Proof. See Stark [16, Lemma 3].

Lemma 2.2. Let $h>1$. Then $a_{2}$ is prime and $a_{2}=\min \left\{p\right.$ prime $\left.\mid\left(\frac{-d}{p}\right) \neq-1\right\}$.

Proof. The integer $a_{2}$ is prime, otherwise choose $p$ prime, $p \mid a_{2}, p<a_{2}$. The number of representations of $p$ with forms with discriminant $-d$ is

$$
R(p)=\sum_{t \mid p}\left(\frac{-d}{t}\right)=1+\left(\frac{-d}{p}\right)=1+\left(\frac{b_{2}^{2}-4 a_{2} c_{2}}{p}\right)=1+\left(\frac{b_{2}^{2}}{p}\right) \geq 1
$$

From Lemma 2.1 only the principal form can represent $p$. This can happen only when $y \neq 0$. But then by Lemma 2.1

$$
\frac{d}{4} \leq p<a_{2} \leq \sqrt{\frac{d}{3}}
$$

consequently $d<\frac{16}{3}$ and $h=1$. This is a contradiction.

Now $a_{2}$ is represented (by $f_{2}$ ), so $\left(\frac{-d}{a_{2}}\right) \neq-1$. Let $p$ be prime, $p<a_{2},\left(\frac{-d}{p}\right) \neq$ -1 . As in (1), $R(p) \geq 1$, but because of $(2), p$ cannot be represented. Contradiction.

Lemma 2.3 (Heilbronn). Let $-d<0$ be a discriminant, $h=h(-d), p$ a rational prime number and $\left(\frac{-d}{p}\right)=1$. Then $p \geq\left(\frac{d}{4}\right)^{\frac{1}{h}}$.

Lemma 2.4 (Heilbronn). Let $-d<0$ be a discriminant, $a>0$ squarefree, $a \mid d$ and $a \leq\left(\frac{d}{4}\right)^{\frac{1}{2}}$. Then there is exactly one reduced form with discriminant $-d$ and leading coefficient $a$. 
2.1. Odd class number. Suppose $h=2 n+1$ with $n \in \mathbb{N}_{0}$. This is the simplest case. By the theory of genera $\left(2^{t-1} \mid h\right.$, where $t$ is the number of distinct prime divisors of $d),-d$ is a prime discriminant, thus has the form $-4,-8$ or $-p$ with $p \equiv 3(\bmod 4)$ prime. It is easy to calculate $h(-4)=h(-8)=1$; we confine our analysis to the discriminants with $d \equiv 3(\bmod 4)$ prime. We will see that the coefficients of the reduced forms behave well.

Lemma 2.5. Let $h>1$ be odd, $f(x, y)=a x^{2}+b x y+c y^{2}$ be a reduced form other than the principal form with discriminant $-d=b^{2}-4 a c$. Then $0<|b|<a<c$.

Proof. We have $b \neq 0$, because $d$ is odd. If $|b|=a$, then $-d=a(a-4 c)$. With $a>1$ and $d$ prime, this leads to $a=d$, a contradiction. If $a=c$, then $b \geq 0$ and $-d=(b-2 a)(b+2 a)$. This leads to $b+2 a=d$ and $b-2 a=-1$, so $b=2 a-1>a$, a contradiction.

Remark 2.6. Let $h>1$ be odd, $f(x, y)=a x^{2}+b x y+c y^{2}$ with $0<|b|<a<c$. Then obviously $a x^{2}-b x y+c y^{2}$ is reduced too. So besides the principal form there are respectively pairs of reduced forms, which differ only by the sign of $b$ (but are nonequivalent).

Corollary 2.7. Let $h>1$ be odd. Then $a_{2}=a_{3}$ and $a_{2}, a_{3}<a_{4}$, i.e., there is exactly one pair of forms with $a_{2}$ as leading coefficient.

Proof. The equalities $a_{3}=a_{4}\left(=a_{5}\right)$ lead to $R\left(a_{2}\right) \geq 4$ (each form represents with $x=1, y=0)$. But because of Lemma 2.2 and $\left(a_{2}, d\right)=1$ we have $R\left(a_{2}\right)=$ $1+\left(\frac{-d}{a_{2}}\right)=2$.

Lemma 2.8. Let $h>1$ be odd. Then $a_{2} \geq\left(\frac{d}{4}\right)^{\frac{1}{h}}$.

Proof. From Lemma 2.2 and Lemma 2.3, because $\left(a_{2}, d\right)=1$.

Lemma 2.9. Let $h>3$ be odd. Then $a_{4}$ is prime or $a_{4}=a_{2}^{n}$ with $1<n \leq \frac{h}{2} \frac{\log \frac{d}{3}}{\log \frac{d}{4}}$.

The proofs of this lemma and of Lemma 2.11 are variations of the proof given for Lemma 2.13 and are therefore omitted. The upper bound for $n$ can be obtained with the help of Lemma 2.8. We also omit the proof of Corollary 2.10, which works like the one for Corollary 2.7.

Corollary 2.10. Let $h=5$ or $h=7$. Then $a_{4}=a_{5}$ and in case of $h=7$, $a_{4}, a_{5}<a_{6}$, i.e., there is exactly one pair of forms with $a_{4}$ as leading coefficient.

Lemma 2.11. Let $h=7$. Then $a_{6}$ is prime or $a_{6}=a_{2}^{n} a_{4}^{m}$ with $n, m \in \mathbb{N}_{0}$, $1<n+m<4$.

We can sum up the results of the preceding lemmas in a way which is useful for us later.

Lemma 2.12. 1) In the case of $h=5$ we have $1<a_{2}=a_{3}<a_{4}=a_{5}$, in the case of $h=7$ we have $1<a_{2}=a_{3}<a_{4}=a_{5}<a_{6}=a_{7}$.

2) Suppose $a_{2}$ has a lower bound $\lambda \in \mathbb{R}$. Let $p_{2}<p_{4}<p_{6}$ be the three least prime numbers $\geq \lambda$. Then in the case of $h=5$ or $h=7, a_{2} \geq p_{2}$ and $a_{4} \geq p_{4}$; moreover, in the case of $h=7$ and $\lambda \geq 4, a_{6} \geq p_{6}$. 
Proof. 1) From 2.6, 2.7 and 2.10.

2) We have $a_{2} \geq p_{2}$ because $a_{2}$ is prime by Lemma 2.2. Now by Lemma 2.9 and 1$), a_{4} \geq p_{4}$ or $a_{4} \geq a_{2}^{2}$. Bertrand's postulate yields a prime number $p, a_{2}<$ $p<2 a_{2} \leq a_{2}^{2}$, hence it is safe to take the first bound. Finally, in case $h=7$, by Lemma 2.11 and 1), $a_{6} \geq p_{6}$ or $a_{6} \geq a_{2}^{2}$. Bertrand's postulate yields for $a_{2} \geq 4$ two prime numbers $p, q, a_{2}<p<q<4 a_{2} \leq a_{2}^{2}$, so it is safe to take the first bound. 2.8).

The condition $\lambda \geq 4$ is satisfied except for very small discriminants (cf. Lemma

2.2. Even class number. Suppose (restrictively) that $h=2 n$ with $n$ odd (e.g., $h=6$ ); then by the theory of genera the number $t$ of distinct prime divisors of $d$ equals 2 . This is because on the one hand, $2^{t-1} \mid h$, so $t \leq 2$, and on the other hand, $h$ is always odd for discriminants with $t=1$ (prime discriminants). Further, take into consideration that $-d \equiv 1(\bmod 4)$, or $-d \equiv 0(\bmod 4)$ and $-\frac{d}{4} \equiv 2,3$ $(\bmod 4)$; then $d$ must have one of the forms

$$
d= \begin{cases}4 p & \text { with } p \text { prime } p \equiv 1 \quad(\bmod 4) \\ 8 p & \text { with } p \text { prime, } p>2 \\ p q & \text { with } p, q \text { prime }, p \equiv 1 \quad(\bmod 4), q \equiv 3 \quad(\bmod 4)\end{cases}
$$

When the class number is even, there are, unlike for odd class number, not necessarily pairs of reduced forms (see Lemma 2.4). For the moment, we collect all reduced forms with $a_{2}$ as leading coefficient under the term $f_{2}$.

Lemma 2.13. Let $h \geq 4$ be even, $f(x, y)=a x^{2}+b x y+c y^{2}$ be a reduced form with $-d=b^{2}-4 a c$ and $a>a_{2}$. Suppose further that the leading coefficients of all reduced forms other than the principal form and $f_{2}$ with discriminant $-d$ are $\geq a$. Then $a$ is prime or $a=a_{2}^{n}$ with $n>1$.

Proof. Let $d^{\prime}=\frac{d}{a_{2}}(\in \mathbb{Q})$. Because $a_{2} \leq\left(\frac{d}{3}\right)^{\frac{1}{2}}=a_{2}^{\frac{1}{2}}\left(\frac{d^{\prime}}{3}\right)^{\frac{1}{2}}$ we have $a_{2}^{\frac{1}{2}} \leq\left(\frac{d^{\prime}}{3}\right)^{\frac{1}{2}}$. Suppose $a$ is not prime, say $p \mid a, p<a, p$ prime. Like in $(1), R(p) \geq 1$. By Lemma 2.1 at most $f_{1}$ and $f_{2}$ can represent $p$. Like in (2) (with $a$ instead of $a_{2}$ ), $f_{1}$ does not represent $p$, therefore $f_{2}$ must represent $p$. Here, with Lemma 2.1, $p=a_{2}$ or $p \geq c_{2}$. Thus, $a$ has the form $a_{2}^{n} p_{1} \cdots p_{m}$ with $n, m \in \mathbb{N}_{0}, p_{i}$ prime, $p_{i} \geq c_{2} \geq \frac{d}{4 a_{2}}=\frac{d^{\prime}}{4}, i=1, \ldots, m$, and so

$$
\frac{d^{\prime}}{3} \geq a_{2}^{\frac{1}{2}}\left(\frac{d^{\prime}}{3}\right)^{\frac{1}{2}}=\left(\frac{d}{3}\right)^{\frac{1}{2}} \geq a \geq a_{2}^{n}\left(\frac{d^{\prime}}{4}\right)^{m} .
$$

If $m \geq 2$, then $\frac{d^{\prime}}{3} \geq\left(\frac{d^{\prime}}{4}\right)^{2}, d^{\prime} \leq \frac{16}{3}$ and $a_{2}=1$. This is a contradiction. If $m=1$, then $n \geq 1$, otherwise $a$ is prime, which is contrary to the supposition. So $\frac{d^{\prime}}{3} \geq a_{2} \frac{d^{\prime}}{4}$ and $a_{2}=1$. This is a contradiction.

Lemma 2.14. Let $h=6$.

1) Suppose $a_{2} \nmid d$. Then $a_{2} \geq\left(\frac{d}{4}\right)^{\frac{1}{6}}$ and $a_{3}=a_{2}$.

2) Suppose $a_{2} \mid d$. Then $a_{3}$ is prime, $a_{3} \geq\left(\frac{d}{4}\right)^{\frac{1}{6}}$. Moreover, if $a_{2}<\left(\frac{3}{16} d\right)^{\frac{1}{2}}$, then $a_{4}=a_{3}$.

Proof. 1) By Lemma 2.2, $\left(\frac{-d}{a_{2}}\right) \neq-1$; since $a_{2} \nmid d$, we have $\left(\frac{-d}{a_{2}}\right) \neq 0$; hence, $\left(\frac{-d}{a_{2}}\right)=1$. Here, on the one hand, $a_{2} \geq\left(\frac{d}{4}\right)^{\frac{1}{6}}$ by Lemma 2.3 , and on the other 
hand, the number of representations $R\left(a_{2}\right)=1+\left(\frac{-d}{a_{2}}\right)=2$. One form with leading coefficient $a_{2}$ yields only one representation. The form $f_{1}$ cannot represent $a_{2}$ (cf. (2)). If $a_{2}<a_{3}$, then the remaining forms cannot do so either (cf. Lemma 2.1).

2) We have $\left(\frac{-d}{a_{2}}\right)=0$, so $R\left(a_{2}\right)=1$, hence $a_{3}>a_{2}$. By Lemma $2.13, a_{3}$ is prime or $a_{3}=a_{2}^{n}$ with $n>1$. The latter yields $R\left(a_{3}\right)=\sum_{t \mid a_{3}}\left(\frac{-d}{t}\right)=\sum_{i=0}^{n}\left(\frac{-d}{a_{2}}\right)^{i}=1$. Consequently, $a_{4}>a_{3}$ and in $f_{3}(x, y)=a_{3} x^{2}+b_{3} x y+c_{3} y^{2}$ it must be that $b_{3}=0$ or $b_{3}=a_{3}$ (otherwise there are two forms with $a_{3}$, and $a_{4}=a_{3}$, cf. the remark to Lemma 2.5). Both cases result in a contradiction: $b_{3}=0$ gives $-d=-4 a_{2}^{n} c_{3}$, $b_{3}=a_{3}$ gives $-d=a_{2}^{n}\left(a_{2}^{n}-4 c_{3}\right)$, and $-d$ is no discriminant. So $a_{3}$ must be prime. Because $a_{2}$ already divides the discriminant, $a_{3}$ cannot do the same, since otherwise, depending on the form of the discriminant (see the beginning of this section) and with the help of the inequalities $d \leq\left(\frac{d}{3}\right)^{\frac{1}{2}}\left(\frac{d}{3}\right)^{\frac{1}{2}}$ resp. $d \leq 8\left(\frac{d}{3}\right)^{\frac{1}{2}}$, we get a contradiction. Hence, $\left(\frac{-d}{a_{3}}\right)= \pm 1$. Furthermore, $\left(\frac{-d}{a_{3}}\right) \neq-1$, because $a_{3}$ is trivially represented. Now Lemma 2.3 gives the second part of the assertion.

Finally let $a_{2}<\left(\frac{3}{16} d\right)^{\frac{1}{2}}$. We have $R\left(a_{3}\right)=2$ like above. One form with leading coefficient $a_{3}$ yields only one representation. The form $f_{1}$ cannot represent $a_{3}$ (cf. (2)). If $a_{3}<a_{4}$, at most a reduced form with $a_{2}$ as leading coefficient can represent $a_{3}$ (cf. Lemma 2.1). But then (cf. Lemma 2.1), $\left(\frac{d}{3}\right)^{\frac{1}{2}} \geq a_{3} \geq c_{2} \geq \frac{d}{4 a_{2}}$ and $a_{2} \geq\left(\frac{3}{16} d\right)^{\frac{1}{2}}$. This is a contradiction.

Corollary 2.15. Let $h=6$. Then $a_{2}$ and $a_{3}$ are prime, and $a_{3} \geq\left(\frac{d}{4}\right)^{\frac{1}{6}}$.

2.3. Integers having prescribed quadratic character. A result of D. H. Lehmer, E. Lehmer and D. Shanks [10] can be used to obtain effective lower bounds for discriminants with "small" class number and "big" leading coefficients of the associated binary quadratic forms. We will use this in $\S 3$ for class number 5 .

Lemma 2.16. Let $p$ be a prime, $p>2$. Define

$$
M_{p}=\min \left\{n \in \mathbb{N} \mid\left(\frac{-n}{q}\right)=-1 \text { for all } q \text { prime, } 2 \leq q<p\right\} .
$$

Then $a_{2} \geq p$ implies $d \geq M_{p}$.

If $d$ is a prime, then in the definition of $M_{p}$ we restrict $n$ to be prime, thus obtaining possibly greater values of $M_{p}$, i.e., better lower bounds for $d$.

Proof. Let $a_{2} \geq p>2$. Recall that by Lemma $2.2, a_{2}$ is prime and $\left(\frac{-d}{q}\right)=-1$ for all prime $q, 2 \leq q<a_{2}$. Therefore, $d \geq M_{a_{2}}$. The inequality $M_{a_{2}} \geq M_{p}$ holds trivially.

The prime values for $M_{p}$ we will use are $M_{131}=193310265163, M_{137,139}=$ $229565917267, M_{149}=915809911867$ and $M_{191}=30059924764123$. They are taken from D. H. Lehmer, E. Lehmer and D. Shanks [10]. It may be noted that our $M_{p}$ equals Lehmer's $N_{q}$, if $q$ is the greatest prime less than $p$. The value for $N_{149}$ had to be corrected, see [16]. The number $M_{191}$ is $N_{181}$ of [15].

\section{ENCIRCLING THE RANGE}

We apply the methods of Stark and Montgomery-Weinberger to "midsized" discriminants. First, we restrict the size of $d$ with the help of 
Theorem 3.1. Let $-d<0$ be a discriminant with class number $h$. Then

$$
h>\frac{1}{55} \prod_{p \mid d} *\left(1-\frac{[2 \sqrt{p}]}{p+1}\right) \log d,
$$

where the * indicates that the greatest prime divisor of $d$ must be omitted.

This theorem originates from the works of D. Goldfeld [4] in 1976, B. Gross and D. Zagier [7] in 1983, and J. Oesterlé [12, 13] in 1984.

Lemma 3.2. Let $-d<0$ be a discriminant. If $h=5$, then $d<10^{120}$; if $h=6$, then $d<10^{574}$; if $h=7$, then $d<10^{168}$.

Proof. If $h=5$ or $h=7$, then $d$ is prime (cf. $\S 2.1$ ). If $h=6$, then $d$ has exactly two distinct prime divisors (cf. $\S 2.2$ ). Now use Theorem 3.1.

Now we turn to $d$ with order of magnitude up to $10^{11} \ldots 10^{14}$ (depending on the class number). They are searched with the use of a computer. It would be very timeconsuming, for example, to examine all discriminants in the range $1 \leq d \leq 10^{11}$ for class number 5. However, Lemma 2.3 helps to reduce the work. Let $p$ be a prime and $d>4 p^{h}$. Then by Lemma 2.3 we have $\left(\frac{-d}{q}\right) \neq 1$ for all prime $q, 2 \leq q \leq p$. This means that in a first step we can discard a certain quantity of $d$ 's by looking at their quadratic character modulo small primes. In a second step we examine the remaining $d$ for class number $h$ by searching for reduced solutions $(-a<b \leq a<c$ or $0 \leq b \leq a=c$ ) of $-d=b^{2}-4 a c$.

Thus, in the range $1 \leq d \leq 1.33 \cdot 10^{11}$ we obtain the $d$-values for $h=5$ as listed in Table 1. Using results of $\S 2.3$, we can slightly raise the bound $1.33 \cdot 10^{11}$ : for $d>1.33 \cdot 10^{11}$, by Lemma 2.8 , we have $a_{2} \geq 131$. By Lemma 2.16 this leads to $d \geq M_{131} \geq 1.9331 \cdot 10^{11}$. Repeating this argument, we get sucessively $a_{2} \geq 139$, $d \geq M_{139} \geq 2.2956 \cdot 10^{11}, a_{2} \geq 149, d \geq M_{149} \geq 9.1580 \cdot 10^{11}, a_{2} \geq 191, d \geq$ $M_{191} \geq 3.0059 \cdot 10^{13}$.

Lemma 3.3. Suppose $h(-d)=5$; then $d$ is one of the numbers listed in Table 1 or $d>3 \cdot 10^{13}$.

In the case of $h=6$ and $h=7, \S 2.3$ cannot be used to reduce the range.

Lemma 3.4. Suppose $h(-d)=7$; then $d$ is one of the numbers listed in Table 1 or $d>8 \cdot 10^{12}$.

Lemma 3.5. Suppose $h(-d)=6$; then $d$ is one of the numbers listed in Table 1 or in case of

1) $17923 \nmid d, a_{2}=2 ; d>6.2 \cdot 10^{13}$,

2) $17923 \nmid d, a_{2}=3 ; d>3 \cdot 10^{13}$,

3) $17923 \nmid d, a_{2} \geq 5 ; d>2 \cdot 10^{13}$,

4) $17923 \mid d ; d>1.1 \cdot 10^{14}$.

We omit the programming details here and refer to [17]. The programs ran about 5 minutes for $h=5,55$ hours for $h=6$, and 11 hours for $h=7$ on the IBM 3090 of the University of Freiburg i. Br. For control purposes the programs were also run on the CRAY 2 of the University of Stuttgart.

The rest of the paper deals with the remaining "midsized" $d$. 


\section{Basics of Stark's Method}

Stark (for details, see [16]) starts from the following representation of the zeta function $\zeta_{\mathbb{K}}(s)=\zeta(s) L\left(s, \chi_{-d}\right)$ of $\mathbb{K}=\mathbb{Q}(\sqrt{-d})$,

$$
\begin{aligned}
\zeta(s) L\left(s, \chi_{-d}\right)= & \zeta(2 s) \sum_{f} a^{-s}+\zeta(2-2 s) \frac{\Gamma(1-s)}{\Gamma(s)}\left(\frac{d}{4 \pi^{2}}\right)^{\frac{1}{2}-s} \sum_{f} a^{s-1} \\
& +\sum_{f} h(s, f) .
\end{aligned}
$$

The summation is over a complete system of nonequivalent binary quadratic forms $f=f(x, y)=a x^{2}+b x y+c y^{2}$ with discriminant $b^{2}-4 a c=-d$. The error terms $h(s, f)$ are

$$
h(s, f)=a^{-s} \sum_{j=1}^{\infty} \int_{-\infty}^{\infty}\left(x-[x]-\frac{1}{2}\right) \frac{d}{d x}\left\{\left(\left(x+\frac{b j}{2 a}\right)^{2}+\frac{d j^{2}}{4 a^{2}}\right)^{-s}\right\} d x .
$$

For these error terms, Stark [16] gave estimates. We adopt them with slight modifications.

Lemma 4.1. Let $s=\sigma+i \tau, \sigma \geq \frac{1}{2}$ and $k \in \mathbb{N}, k \geq 3$. Then

$$
|h(s, f)|<2\left(\frac{4 a}{d}\right)^{\sigma-\frac{1}{2}}\left(\frac{2 \pi}{a k}\right)^{\frac{1}{2}}\left(\frac{k-1}{k-2}\right)\left(\frac{2|s|+\frac{k-1}{2}}{\pi\left(\frac{d}{a^{2}}\right)^{\frac{1}{2}}}\right)^{k} \exp \left(\frac{1}{4 k}+\frac{\sqrt{2}}{3 \pi^{3} k^{2}}\right) .
$$

Proof. See Stark [16, Lemma 1]. Simply change the estimate for $\zeta(m)$ to $\zeta(m)<$ $\sqrt{(m-1) /(m-2)} \quad(m \geq 3)$.

Lemma 4.2. Let $a \leq\left(\frac{d}{3}\right)^{\frac{1}{2}}$ and $s=\frac{1}{2}+i \tau$. Then for all $J \in \mathbb{N}$

$$
|h(s, f)|<\frac{4|s|}{(3 d)^{\frac{1}{4}}}\left(\sum_{j=1}^{J-1} \frac{1}{j}+\frac{\frac{2}{3} \tau+0.77}{3 \sqrt{3}} \sum_{j=J}^{\infty} \frac{1}{j^{2}}\right) .
$$

Proof. See Stark [16, Lemma 2]. We do not use the estimate for

$$
\left|(2 s+1)-\frac{2 s+2}{u^{2}+1}\right| ;
$$

instead, we let $s=\frac{1}{2}+i \tau$ in (5) and obtain

$$
\begin{aligned}
& \left|\int_{-\infty}^{\infty}\left(x-[x]-\frac{1}{2}\right) \frac{d}{d x}\left\{\left(\left(x+\frac{b j}{2 a}\right)^{2}+\frac{d j^{2}}{4 a^{2}}\right)^{-s}\right\} d x\right| \\
& \quad \leq \frac{4 a^{2}|s|}{3 d j^{2}} \int_{0}^{\frac{\pi}{2}} \sqrt{\left(\left(2-3 \cos ^{2} x\right) \cos x\right)^{2}+\left(2 \tau \sin ^{2} x \cos x\right)^{2}} d x \\
& \quad \leq \frac{4 a^{2}|s|}{3 d j^{2}}\left(\frac{4}{3} \sin \left(\arccos \sqrt{\frac{2}{3}}\right)+\frac{2}{3} \tau\right) .
\end{aligned}
$$




\section{Basics of Montgomery-Weinberger}

Let $-k<0$ be a discriminant with $(d, k)=1$. Further, let $\theta$ be a complex number with $|\theta| \leq 1$, the value of which depends on the context in which it occurs, e.g., $\theta$ in Lemma 5.1 and $\theta$ in Lemma 5.3 need not be the same. Montgomery and Weinberger [11] proceed from

Lemma 5.1. Let $(d, k)=1, t \geq 0$. Then

(7)

$$
\text { it } L\left(\frac{1}{2}+i t, \chi_{k}\right) L\left(\frac{1}{2}+i t, \chi_{k d}\right) \Gamma\left(\frac{1}{2}+i t\right)\left(\frac{k d^{\frac{1}{2}}}{2 \pi}\right)^{i t}=i M(t) \sin \varphi(t)+\theta t E(t),
$$

in which

$$
\begin{gathered}
M(t)=\left|2 t \zeta(1+2 i t) \Gamma\left(\frac{1}{2}+i t\right) P_{k}\left(\frac{1}{2}+i t\right) A\left(\frac{1}{2}+i t\right)\right| \\
\varphi(t)=\arg \left(i \zeta(1+2 i t) \Gamma\left(\frac{1}{2}+i t\right) P_{k}\left(\frac{1}{2}+i t\right) A\left(\frac{1}{2}+i t\right)\left(\frac{k d^{\frac{1}{2}}}{2 \pi}\right)^{i t}\right),
\end{gathered}
$$

with

$$
P_{k}(s)=\prod_{p \mid k}\left(1-p^{-2 s}\right), \quad A(s)=\sum_{f} \chi_{k}(a) a^{-s}
$$

and

$$
E(t)=\frac{4 \pi^{\frac{1}{2}}}{k} \sum_{f} a^{-\frac{1}{2}} \sum_{n=1}^{\infty} K_{0}\left(\frac{\pi n d^{\frac{1}{2}}}{a k}\right) \sum_{y \mid n}\left|\sum_{j=1}^{k} \chi_{k}(f(j, y)) \exp \left(\frac{2 \pi i j n}{k y}\right)\right|,
$$

where $K_{0}$ is the modified Bessel function of the second kind (note that $E(t) \geq 0$ ).

If in (7) we let $t$ be the imaginary part of a zero $\frac{1}{2}+i t$ of $L\left(s, \chi_{k}\right)$, then

$$
|\sin \varphi(t)| \leq \frac{t E(t)}{M(t)}
$$

This relation, with sufficiently large $d$ and appropriate choice of $k$ and $t$, may be used to get a contradiction to the assumption of a small class number. The following lemmas provide estimates for $M(t), \varphi(t)$ and $E(t)$. For proofs see [11].

Lemma 5.2. Let $0<t \leq \frac{1}{20}$. Then $M(t) \geq \frac{7}{4} \prod_{p \mid k}\left(1-p^{-1}\right)\left|A\left(\frac{1}{2}+i t\right)\right|$.

Lemma 5.3. Let $0 \leq t \leq 6$. Then

$$
\varphi(t)=t\left(C+\log \left(\frac{k d^{\frac{1}{2}}}{8 \pi}\right)\right)+3 \theta t^{3}+\theta t\left(c(h)+2 \sum_{p \mid k} \frac{\log p}{p-1}\right),
$$

in which $C=0.577215664 \ldots$ is Euler's constant and $c(h) \leq\left|\frac{A^{\prime}}{A}\left(\frac{1}{2}+i t\right)\right|$.

Lemma 5.4. Let $k \geq 3060,0<t \leq \frac{1}{4}$. Define

$$
\delta(a)=\left(\frac{a}{d}\right)^{\frac{1}{2}} \exp \left(-\frac{\pi d^{\frac{1}{2}}}{2 a k}\right) .
$$


Then, if all forms $f$ are reduced,

$$
E(t) \leq 8\left(\frac{k}{\pi}\right)^{\frac{1}{2}} \log k \prod_{p \mid k}\left(2+3 p^{-\frac{3}{2}}\right) \sum_{f} \delta(a) .
$$

Proof. While proving Lemma 9 of [11], Montgomery and Weinberger get

$$
E(t) \leq 4\left(\frac{\pi}{k}\right)^{\frac{1}{2}} \prod_{p \mid k}\left(2+3 p^{-\frac{3}{2}}\right) \sum_{f} a^{-\frac{1}{2}} g\left(\frac{\pi d^{\frac{1}{2}}}{2 a k}\right),
$$

with $g(x)=\frac{e^{-x}}{x}\left(1+\log \left(1+\frac{1}{x}\right)\right)$. So the summands of the sum over $f$ have the form

$$
\frac{2 a^{\frac{1}{2}} k}{\pi d^{\frac{1}{2}}} \exp \left(-\frac{\pi d^{\frac{1}{2}}}{2 a k}\right)\left(1+\log \left(1+\frac{2 a k}{\pi d^{\frac{1}{2}}}\right)\right) .
$$

The third factor, by means of the inequality $a \leq \sqrt{\frac{d}{3}}$, is bounded uniformly for all $a$ by $\log k$ (for $k \geq 3060$ ).

If $a$ is not effectively known, we will use the last lemma either under the (nontrivial) assumption $a \leq\left(\frac{d}{4}\right)^{\frac{1}{3}}$ or under the (trivial) assumption $a \leq\left(\frac{d}{3}\right)^{\frac{1}{2}}$ in $\delta(a)$.

\section{Class Number 5 And 7}

In case of an odd class number both Stark's and Montgomery-Weinberger's method can be applied. In [17] we used the first method for class number 5 and the second method for class number 7. However, the adaption of Stark's method was somewhat more tedious, so for brevity here we proceed like in [11]. This is straightforward; therefore, we can confine ourselves to a short survey. Details on working with Montgomery-Weinberger's method appear in $\S 7$.

For $h=5$ we examine the range $2 \cdot 10^{12} \leq d \leq 10^{52}$ and distinguish three cases for the leading coefficients $a_{2}\left(=a_{3}\right)$ and $a_{4}\left(=a_{5}\right)$ of the reduced forms by comparing them with $\left(\frac{d}{4}\right)^{\frac{1}{3}}$. If the $a_{i}$ are "small", then Lemma 5.4 will give us a "good" bound for $E(t)$. If the $a_{i}$ are "big", then the bounds for $\left|A\left(\frac{1}{2}+i t\right)\right|$ and $c(5)$ in Lemmas 5.2 and 5.3 will be "good". In all cases we use $k=17923$ and $t=0.030986$ (see Table 2). Finally, we examine $d$ in the range $10^{52} \ldots 10^{120}$, using $k=115147$ and $t=0.003158$. The result is

Theorem 6.1. Suppose $2 \cdot 10^{12} \leq d \leq 10^{120}$. Then $h(-d) \neq 5$.

TABLE 2. Zeros $\frac{1}{2}+i t$ of $L\left(s, \chi_{k}\right)$ for various $k$; from [11]

\begin{tabular}{||r|r|r||}
\hline \multicolumn{1}{||c|}{$k$} & $t+10^{-6} \theta$ \\
\hline \hline 17923 & & 0.030986 \\
28963 & $=11 \cdot 2633$ & 0.033774 \\
30895 & $=5 \cdot 37 \cdot 167$ & 0.018494 \\
37427 & $=13 \cdot 2879$ & 0.019505 \\
115147 & $=113 \cdot 1019$ & 0.003158 \\
123204 & $=4 \cdot 3 \cdot 10267$ & 0.010650 \\
139011 & $=3 \cdot 46337$ & 0.012930 \\
\hline
\end{tabular}


For $h=7$ we examine the range $8 \cdot 10^{12} \leq d \leq 10^{168}$ and distinguish four cases for the leading coefficients $a_{2}\left(=a_{3}\right), a_{4}\left(=a_{5}\right), a_{6}\left(=a_{7}\right)$ of the reduced forms, completely analogous to $h=5$. We get

Theorem 6.2. Suppose $8 \cdot 10^{12} \leq d \leq 10^{168}$. Then $h(-d) \neq 7$.

\section{Class number 6}

7.1. Results with Montgomery-Weinberger. We first turn to $d \leq 10^{52}$ and distinguish certain cases for the coefficients $a_{2}, a_{3}, \ldots, a_{6}$ (like we did for $h=5,7$ ). The problem is that $a_{2}$ no longer needs to be relatively prime to $d$, and therefore the lower bound of Lemma 2.8 will fail. We distinguish the cases $17923 \nmid d$ and $17923 \mid d$. In the first case we further distinguish $a_{2}=2, a_{2}=3,5 \leq a_{2}<\left(\frac{d}{4}\right)^{\frac{1}{6}}$ and $a_{2} \geq\left(\frac{d}{4}\right)^{\frac{1}{6}}$, and in the latter case $a_{2}=17923$ and $a_{2}<17923$.

1. $17923 \nmid d$

$\mathbf{1}$ (a) $a_{2}=2$

Here we restrict our argument to $d$ being even, because if $d$ is odd, then by Lemmas 2.2 and $2.3,2 \geq\left(\frac{d}{4}\right)^{\frac{1}{6}}$. Further, by Lemma 2.14 we have $a_{3}$ prime, $a_{3} \geq$ $\left(\frac{d}{4}\right)^{\frac{1}{6}}$ and $a_{4}=a_{3}$.

Lemma 7.1. Let $17923 \nmid d, a_{2}=2$, and suppose $2.3 \cdot 10^{14} \leq d \leq 10^{52}, a_{i} \geq\left(\frac{d}{4}\right)^{\frac{1}{3}}$, $i=3, \ldots, 6$. Then $h(-d) \neq 6$.

Proof. A sample proof is given in the next lemma. We have $a_{i} \geq 38598, i=3, \ldots, 6$. Take $k=17923$ and $t=0.030986$.

Lemma 7.2. Let $17923 \nmid d, a_{2}=2$, and suppose $6.2 \cdot 10^{13} \leq d \leq 10^{52}, a_{i}<\left(\frac{d}{4}\right)^{\frac{1}{3}}$, $i=3,4, a_{i} \geq\left(\frac{d}{4}\right)^{\frac{1}{3}}, i=5,6$. Then $h(-d) \neq 6$.

Proof. We have $a_{3}, a_{4} \geq 163, a_{5}, a_{6} \geq 24934$. Take $k=30895=5 \cdot 37 \cdot 167$ $((d, k)=1$, else $d=(4 p$ or $8 p) \leq 8 \cdot 167)$ and $t=0.018494$. Then

$$
\left|A\left(\frac{1}{2}+i t\right)\right| \geq \underbrace{|1+\underbrace{\left(\frac{-30895}{2}\right)}_{=1} 2^{-\frac{1}{2}-i t}|}_{\geq 1.707}-\frac{2}{\sqrt{163}}-\frac{2}{\sqrt{24934}},
$$

and thus $M(t) \geq 2.0820$ by Lemma 5.2 ,

$$
c(6) \leq \frac{\frac{\log 2}{\sqrt{2}}+2 \frac{\log 163}{\sqrt{163}}+2 \frac{\log 24934}{\sqrt{24934}}}{1.707-\frac{2}{\sqrt{163}}-\frac{2}{\sqrt{24934}}} \leq 0.9211
$$

and $E(t) \leq 43.5542$ by Lemma $5.4,|\sin \varphi(t)| \leq 0.3869$ by $(8)$. But $0.3991 \leq \varphi(t) \leq$ 1.2863 by Lemma 5.3 .

Lemma 7.3. Let $17923 \nmid d, a_{2}=2$, and suppose $6.2 \cdot 10^{13} \leq d \leq 10^{52}$ and

1) $a_{i}<\left(\frac{d}{4}\right)^{\frac{1}{3}}, i=3,4,5, a_{6} \geq\left(\frac{d}{4}\right)^{\frac{1}{3}}$ or

2) $a_{i}<\left(\frac{d}{4}\right)^{\frac{1}{3}}, i=3, \ldots, 6$.

Then $h(-d) \neq 6$.

Proof. In both cases take $k=30895$. 
In $\S 7.2$ we will use Stark's method to close the gap $6.2 \cdot 10^{13} \leq d \leq 2.3 \cdot 10^{14}$, which was left by Lemma 7.1; so for all $d$ with $a_{2}=2$ we will have the uniform bound $6.2 \cdot 10^{13}$.

1(b) $a_{2}=3$

Here we have $($ for $d>2916) d \equiv 0(\bmod 3)$ by Lemmas 2.2 and 2.3. Further, by Lemma 2.14 we have $a_{3}$ prime, $a_{3} \geq\left(\frac{d}{4}\right)^{\frac{1}{6}}$ and $a_{4}=a_{3}$.

Lemma 7.4. Let $17923 \nmid d, a_{2}=3$, and suppose $3 \cdot 10^{13} \leq d \leq 10^{52}$ and

1) $a_{i} \geq\left(\frac{d}{4}\right)^{\frac{1}{3}}, i=3, \ldots, 6$, or

2) $a_{i}<\left(\frac{d}{4}\right)^{\frac{1}{3}}, i=3,4, a_{i} \geq\left(\frac{d}{4}\right)^{\frac{1}{3}}, i=5,6$, or

3) $a_{i}<\left(\frac{d}{4}\right)^{\frac{1}{3}}, i=3,4,5, a_{6} \geq\left(\frac{d}{4}\right)^{\frac{1}{3}}$, or

4) $a_{i}<\left(\frac{d}{4}\right)^{\frac{1}{3}}, i=3, \ldots, 6$.

Then $h(-d) \neq 6$.

Proof. In all cases, let $k=37427=13 \cdot 2879((d, k)=1$, else $d=3 p \leq 3 \cdot 2879)$, $t=0.019505$ and use $\left(\frac{-37427}{3}\right)=1$ to estimate $\left|A\left(\frac{1}{2}+i t\right)\right|$.

$\mathbf{1}(\mathbf{c}) 5 \leq a_{2}<\left(\frac{d}{4}\right)^{\frac{1}{6}}$

Here, $d \equiv 0\left(\bmod a_{2}\right)$ by Lemmas 2.2 and 2.3. Further, by Lemma $2.14, a_{3}$ is prime, $a_{3} \geq\left(\frac{d}{4}\right)^{\frac{1}{6}}$ and $a_{4}=a_{3}$.

Lemma 7.5. Let $17923 \nmid d, 5 \leq a_{2}<\left(\frac{d}{4}\right)^{\frac{1}{6}}$; suppose $2 \cdot 10^{13} \leq d \leq 10^{52}$ and

1) $a_{i} \geq\left(\frac{d}{4}\right)^{\frac{1}{3}}, i=3, \ldots, 6$, or

2) $a_{i}<\left(\frac{d}{4}\right)^{\frac{1}{3}}, i=3,4, a_{i} \geq\left(\frac{d}{4}\right)^{\frac{1}{3}}, i=5,6$, or

3) $a_{i}<\left(\frac{d}{4}\right)^{\frac{1}{3}}, i=3,4,5, a_{6} \geq\left(\frac{d}{4}\right)^{\frac{1}{3}}$, or

4) $a_{i}<\left(\frac{d}{4}\right)^{\frac{1}{3}}, i=3, \ldots, 6$.

Then $h(-d) \neq 6$.

Proof. In all cases, let $k=17923$.

$\mathbf{1}(\mathbf{d}) a_{2} \geq\left(\frac{d}{4}\right)^{\frac{1}{6}}$

Lemma 7.6. Let $17923 \nmid d, 2 \cdot 10^{13} \leq d \leq 10^{52}$ and

1) $a_{2} \geq\left(\frac{d}{4}\right)^{\frac{1}{3}}$, or

2) $\left(\frac{d}{4}\right)^{\frac{1}{6}} \leq a_{2}<\left(\frac{d}{4}\right)^{\frac{1}{3}}$. Then $h(-d) \neq 6$.

Proof. In both cases, take $k=17923$.

2. $17923 \mid d$

Here, $d$ has exactly two distinct prime divisors. Without loss of generality we may assume 17923 to be the smaller one, since otherwise $d<4 \cdot 10^{8}$. Further, assume $17923 \leq\left(\frac{d}{4}\right)^{\frac{1}{2}}$, since otherwise $d<2 \cdot 10^{9}$. Lemma 2.4 yields the existence 
of a reduced form with discriminant $-d$ and 17923 as leading coefficient. Therefore, $a_{2} \leq 17923$.

2(a) $a_{2}=17923$

Lemma 7.7. Let $17923 \mid d, a_{2}=17923$, and suppose $1.1 \cdot 10^{14} \leq d \leq 10^{52}$. Then $h(-d) \neq 6$.

Proof. Take $k=28963=11 \cdot 2633$.

2(b) $a_{2}<17923$

Here, $d \not \equiv 0\left(\bmod a_{2}\right)$, else 17923 is not the smallest prime divisor of $d$. By Lemma 2.14 it follows that $a_{3}=a_{2}$. Both coefficients are prime and $\geq\left(\frac{d}{4}\right)^{\frac{1}{6}}$.

Lemma 7.8. Let $17923 \mid d, a_{2}<17923$; suppose $1.1 \cdot 10^{14} \leq d \leq 10^{52}$ and

1) $a_{i} \geq\left(\frac{d}{4}\right)^{\frac{1}{3}}, i=4,5,6$, or

2) $a_{4}<\left(\frac{d}{4}\right)^{\frac{1}{3}}, a_{i} \geq\left(\frac{d}{4}\right)^{\frac{1}{3}}, i=5,6$, or

3) $a_{i}<\left(\frac{d}{4}\right)^{\frac{1}{3}}, i=4,5, a_{6} \geq\left(\frac{d}{4}\right)^{\frac{1}{3}}$, or

4) $a_{i}<\left(\frac{d}{4}\right)^{\frac{1}{3}}, i=4,5,6$.

Then $h(-d) \neq 6$.

Proof. In all cases, take $k=28963$.

Finally, we consider $d$ from $10^{52}$ to $10^{574}$. It is necessary to examine just two cases.

Lemma 7.9. Suppose $10^{52} \leq d \leq 10^{574}$ and $(d, 115147)=1$. Then $h(-d) \neq 6$.

Proof. Clearly, $a_{2} \geq 2$. By Corollary 2.15 we have $a_{i} \geq\left(\frac{d}{4}\right)^{\frac{1}{6}}, i=3, \ldots, 6$. Take $k=115147=113 \cdot 1019$.

Lemma 7.10. Suppose $10^{52} \leq d \leq 10^{574}$ and $(d, 115147)>1$. Then $h(-d) \neq 6$.

Proof. We have $113 \mid d$ or $1019 \mid d$. But then $(d, 123204=4 \cdot 3 \cdot 10267)=1$, otherwise $d$ is too small. Take $k=123204, t=0.003158$. We have $a_{i} \geq 113$, $i=2, \ldots, 6\left(a_{2}<113 \Rightarrow a_{2}<\left(\frac{d}{4}\right)^{\frac{1}{6}} \Rightarrow a_{2} \mid d \Rightarrow d\right.$ too small; but $a_{2}=113$ is possible, cf. Lemma 2.4). We get $M(t) \geq 0.5282, c(6) \leq 0.4956, E(t) \leq 2 \cdot 10^{-7}$ and $|\sin \varphi(t)| \leq 5 \cdot 10^{-9}$. But $0.7024 \leq \varphi(t) \leq 7.1664$. Therefore, $\varphi=\pi+\theta 10^{-8}$ or $\varphi=2 \pi+\theta 10^{-8}$. By Lemma 5.3 we see that $10^{122}<d<10^{126}$ or $10^{250}<d<10^{254}$ must hold.

However, we can also work with $k=139011=3 \cdot 46337, t=0.003158$. Then we get $M(t) \geq 1.0566, c(6) \leq 0.4956, E(t) \leq 4 \cdot 10^{-8}$ and $|\sin \varphi(t)| \leq 5 \cdot 10^{-10}$. But also $0.8723 \leq \varphi(t) \leq 8.6843$. Therefore, $\varphi=\pi+\theta 10^{-8}$ or $\varphi=2 \pi+\theta 10^{-8}$. By Lemma $5.3,10^{100}<d<10^{103}$ or $10^{206}<d<10^{208}$ must hold. The contradiction of the results for the two $k$ 's proves the lemma.

7.2. Results with Stark's method. We will only examine the case $a_{2}=2$, $a_{i} \geq\left(\frac{d}{4}\right)^{\frac{1}{3}}, i=3, \ldots, 6,6.2 \cdot 10^{13} \leq d \leq 2.3 \cdot 10^{14}$ (remember Lemma 7.1 and 
preceding remarks), which could not be treated with the method of MontgomeryWeinberger. (The condition $17923 \nmid d$ is insignificant here.)

Let $\phi_{m}=\frac{1}{2}+i \tau_{m}$ be a zero of the Riemann zeta function $\zeta(s)$. Putting $\phi_{m}$ in (3) gives

$$
\begin{aligned}
& \left(\frac{d}{4 \pi^{2}}\right)^{i \tau_{m}}\left(1+\frac{\sum_{f} h\left(\frac{1}{2}+i \tau_{m}, f\right)}{\zeta\left(1+2 i \tau_{m}\right)\left(1+2^{-\frac{1}{2}-i \tau_{m}}+2 a_{3}^{-\frac{1}{2}-i \tau_{m}}+a_{5}^{-\frac{1}{2}-i \tau_{m}}+a_{6}^{-\frac{1}{2}-i \tau_{m}}\right)}\right) \\
& =-\frac{\zeta\left(1-2 i \tau_{m}\right)}{\zeta\left(1+2 i \tau_{m}\right)} \frac{\Gamma\left(\frac{1}{2}-i \tau_{m}\right)}{\Gamma\left(\frac{1}{2}+i \tau_{m}\right)} \frac{\left(1+2^{-\frac{1}{2}+i \tau_{m}}+2 a_{3}^{-\frac{1}{2}+i \tau_{m}}+a_{5}^{-\frac{1}{2}+i \tau_{m}}+a_{6}^{-\frac{1}{2}+i \tau_{m}}\right)}{\left(1+2^{-\frac{1}{2}-i \tau_{m}}+2 a_{3}^{-\frac{1}{2}-i \tau_{m}}+a_{5}^{-\frac{1}{2}-i \tau_{m}}+a_{6}^{-\frac{1}{2}-i \tau_{m}}\right)}
\end{aligned}
$$

Define

$$
\begin{gathered}
\alpha_{m} \equiv \pi-2 \arg \zeta\left(2 \phi_{m}\right)-2 \arg \Gamma\left(\phi_{m}\right) \quad(\bmod 2 \pi), \quad 0 \leq \alpha_{m}<2 \pi \\
A_{n}=\frac{1}{2 \pi}\left(\frac{\tau_{n}}{\tau_{1}} \alpha_{1}-\alpha_{n}\right) .
\end{gathered}
$$

Let $|\theta| \leq 1$ as in $\S 5$. For $a_{3} \geq 191$ let

$$
\delta_{m}\left(a_{3}, a_{5}, a_{6}\right)=\frac{\left|h\left(\phi_{m}, f_{1}\right)\right|+\left|h\left(\phi_{m}, f_{2}\right)\right|+2\left|h\left(\phi_{m}, f_{3}\right)\right|+\left|h\left(\phi_{m}, f_{5}\right)\right|+\left|h\left(\phi_{m}, f_{6}\right)\right|}{\left|\zeta\left(2 \phi_{m}\right)\right|\left(\left|1+2^{-\frac{1}{2}-i \tau_{m}}\right|-2 a_{3}^{-\frac{1}{2}}-a_{5}^{-\frac{1}{2}}-a_{6}^{-\frac{1}{2}}\right)} .
$$

Lemma 7.11. Suppose $a_{2}=2, a_{3} \geq 191, \delta_{m}\left(a_{3}, a_{5}, a_{6}\right)<\frac{1}{2}$. Then there is an integer $x_{m}$ with

$$
\begin{aligned}
\tau_{m} \log \left(\frac{d}{4 \pi^{2}}\right)= & \alpha_{m}+2 \pi x_{m} \\
& +2 \arg \left(1+2^{-\frac{1}{2}+i \tau_{m}}+2 a_{3}^{-\frac{1}{2}+i \tau_{m}}+a_{5}^{-\frac{1}{2}+i \tau_{m}}+a_{6}^{-\frac{1}{2}+i \tau_{m}}\right) \\
& +\frac{\pi}{3} \delta_{m}\left(a_{3}, a_{5}, a_{6}\right) \theta
\end{aligned}
$$

Proof. Cf. Stark [16, Lemma 6].

Lemma 7.12. Suppose $a_{2}=2, a_{3} \geq 751, \delta_{m}\left(a_{3}, a_{5}, a_{6}\right)<\frac{1}{2}$ for $m=1$ and $m=n$. Then

$$
\begin{aligned}
x_{n}= & \frac{\tau_{n}}{\tau_{1}} x_{1}+A_{n}+\frac{1}{\pi}\left(\frac{\tau_{n}}{\tau_{1}} \arg \left(1+2^{-\frac{1}{2}+i \tau_{1}}\right)-\arg \left(1+2^{-\frac{1}{2}+i \tau_{n}}\right)\right) \\
& +\frac{\theta}{3}\left(\frac{\tau_{n}}{\tau_{1}} \frac{2 a_{3}^{-\frac{1}{2}}+a_{5}^{-\frac{1}{2}}+a_{6}^{-\frac{1}{2}}}{\left|1+2^{-\frac{1}{2}+i \tau_{1}}\right|}+\frac{2 a_{3}^{-\frac{1}{2}}+a_{5}^{-\frac{1}{2}}+a_{6}^{-\frac{1}{2}}}{\left|1+2^{-\frac{1}{2}+i \tau_{n}}\right|}\right) \\
& +\frac{\theta}{6}\left(\frac{\tau_{n}}{\tau_{1}} \delta_{1}\left(a_{3}, a_{5}, a_{6}\right)+\delta_{n}\left(a_{3}, a_{5}, a_{6}\right)\right) .
\end{aligned}
$$

Proof. Cf. Stark [16, Lemma 7]. However, here we use the relation

$$
\arg \left(1+z+z^{\prime}\right)=\arg (1+z)+\frac{\pi}{3} \theta \frac{\left|z^{\prime}\right|}{|1+z|} \quad \text { for } \frac{\left|z^{\prime}\right|}{|1+z|} \leq \frac{1}{2} .
$$


TABLE 3. $\tau_{m}$ and related quantities; from Stark [16]

\begin{tabular}{||c|c|c|c|c|r||}
\hline$m$ & $\tau_{m}+5 \cdot 10^{-10} \theta$ & $\frac{\alpha_{m}}{2 \pi}+10^{-7} \theta$ & $\left|\zeta\left(2 \phi_{m}\right)\right|+10^{-4} \theta$ & $\frac{\tau_{m}}{\tau_{1}}+5 \cdot 10^{-10} \theta$ & $A_{m}+5 \cdot 10^{-10} \theta$ \\
\hline \hline 1 & 14.134725142 & 0.189940085 & 1.9488 & - & \\
\hline 2 & 21.022039639 & 0.744277023 & 0.8310 & 1.487262004 & -0.461786352 \\
\hline
\end{tabular}

Lemma 7.13. Suppose $a_{2}=2, a_{3} \geq 191$ and $d \geq 20000$. Then

$$
\delta_{m}\left(a_{3}, a_{5}, a_{6}\right)<\left\{\begin{array}{l}
10^{-27}+200.0 d^{-\frac{1}{4}}\left(0.427-2 a_{3}^{-\frac{1}{2}}-a_{5}^{-\frac{1}{2}}-a_{6}^{-\frac{1}{2}}\right)^{-1} \text { for } m=1, \\
10^{-27}+807.4 d^{-\frac{1}{4}}\left(0.951-2 a_{3}^{-\frac{1}{2}}-a_{5}^{-\frac{1}{2}}-a_{6}^{-\frac{1}{2}}\right)^{-1} \text { for } m=2 .
\end{array}\right.
$$

Proof. For $a_{1}=1$ and $a_{2}=2$ we could use Lemma 4.1 (with $\frac{d}{a^{2}} \geq 5000$ ) to get $\left|h\left(\phi_{m}, f\right)\right|<10^{-30}$ for $m \leq 2$ (with $k=137$ ). For $a_{3}, \ldots, a_{6}$ we used Lemma 4.2 to get $\left|h\left(\phi_{m}, f\right)\right|<C d^{-\frac{1}{4}}$ with $C=97.4$ for $m=1$ (with $J=2$ ) and $C=167.7$ for $m=2$ (with $J=3$ ). By direct computation we obtained $\left|1+2^{-\frac{1}{2}-i \tau_{1}}\right| \geq 0.427$, $\left|1+2^{-\frac{1}{2}-i \tau_{2}}\right| \geq 0.951$. See also Table 3 .

Lemma 7.14. Suppose $d>6.2 \cdot 10^{13}, a_{2}=2, a_{3} \geq\left(\frac{d}{4}\right)^{\frac{1}{3}}$. Then

$$
\begin{aligned}
& x_{1}>2.249 \log d-8.543-0.398\left(2^{-\frac{1}{2}}+2 a_{3}^{-\frac{1}{2}}+a_{5}^{-\frac{1}{2}}+a_{6}^{-\frac{1}{2}}\right), \\
& d>41.39 \exp \left(0.444 x_{1}-0.177\left(2^{-\frac{1}{2}}+2 a_{3}^{-\frac{1}{2}}+a_{5}^{-\frac{1}{2}}+a_{6}^{-\frac{1}{2}}\right)\right) .
\end{aligned}
$$

Under the assumptions made, we always have $x_{1} \geq 63$, and if $x_{1} \geq 67$, then $d>2.3 \cdot 10^{14}$.

Proof. Analogous to [16, Lemma 8]. We have $\delta_{1}\left(a_{3}, a_{5}, a_{6}\right)<\frac{1}{2}$ by Lemma 7.13 , because $a_{3}, \ldots, a_{6} \geq 24934$. Now use Lemma 7.11 and the inequality $|\arg (1+z)|<$ $\frac{5}{4}|z|$ for $|z| \leq \frac{9}{10}$.

Lemma 7.15. Suppose $d \geq 6.2 \cdot 10^{13}, a_{2}=2, a_{i} \geq\left(\frac{d}{4}\right)^{\frac{1}{3}}, i=3, \ldots, 6$. Then

$$
\begin{aligned}
\mid x_{2}- & \frac{\tau_{2}}{\tau_{1}} x_{1}-A_{2}-\frac{1}{\pi}\left(\frac{\tau_{2}}{\tau_{1}} \arg \left(1+2^{-\frac{1}{2}+i \tau_{1}}\right)-\arg \left(1+2^{-\frac{1}{2}+i \tau_{2}}\right)\right) \mid \\
< & 10^{-27}+\frac{2 a_{3}^{-\frac{1}{2}}+a_{5}^{-\frac{1}{2}}+a_{6}^{-\frac{1}{2}}}{3}\left(\frac{\tau_{2}}{\tau_{1}} \frac{1}{\left|1+2^{-\frac{1}{2}+i \tau_{1}}\right|}+\frac{1}{\left|1+2^{-\frac{1}{2}+i \tau_{2}}\right|}\right) \\
& +\frac{1}{6} d^{-\frac{1}{4}}\left(\frac{\tau_{2}}{\tau_{1}} \frac{200.0}{0.427-2 a_{3}^{-\frac{1}{2}}-a_{5}^{-\frac{1}{2}}-a_{6}^{-\frac{1}{2}}}+\frac{807.4}{0.951-2 a_{3}^{-\frac{1}{2}}-a_{5}^{-\frac{1}{2}}-a_{6}^{-\frac{1}{2}}}\right) .
\end{aligned}
$$

Proof. Directly from Lemma 7.12 , because $\delta_{1}\left(a_{3}, a_{5}, a_{6}\right)<\frac{1}{2}$ and $\delta_{2}\left(a_{3}, a_{5}, a_{6}\right)<\frac{1}{2}$ by Lemma 7.13.

Lemma 7.16. Suppose $6.2 \cdot 10^{13} \leq d \leq 2.3 \cdot 10^{14}, a_{2}=2, a_{i} \geq\left(\frac{d}{4}\right)^{\frac{1}{3}}, i=3, \ldots, 6$. Then $h(-d) \neq 6$. 
Proof. We have $a_{i} \geq 24934, i=3, \ldots, 6$, and $x_{1} \geq 63$ by Lemma 7.14. We want to show that $x_{1} \geq 67$, for then $d>2.3 \cdot 10^{14}$ by Lemma 7.14 . By Lemma 7.15 we have

$$
\left|x_{2}-\frac{\tau_{2}}{\tau_{1}} x_{1}-A_{2}-\frac{1}{\pi}\left(\frac{\tau_{2}}{\tau_{1}} \arg \left(1+2^{-\frac{1}{2}+i \tau_{1}}\right)-\arg \left(1+2^{-\frac{1}{2}+i \tau_{2}}\right)\right)\right|<0.14 .
$$

But from

$$
\frac{1}{\pi}\left(\frac{\tau_{2}}{\tau_{1}} \arg \left(1+2^{-\frac{1}{2}+i \tau_{1}}\right)-\arg \left(1+2^{-\frac{1}{2}+i \tau_{2}}\right)\right)=-0.5414+10^{-4} \theta
$$

and $\frac{\tau_{2}}{\tau_{1}}=1.487262004+5 \cdot 10^{-10} \theta, A_{2}=-0.461786352+5 \cdot 10^{-10} \theta$ (cf. Table 3 ) it follows that

$$
\left|x_{2}-\frac{\tau_{2}}{\tau_{1}} x_{1}-A_{2}-\frac{1}{\pi}\left(\frac{\tau_{2}}{\tau_{1}} \arg \left(1+2^{-\frac{1}{2}+i \tau_{1}}\right)-\arg \left(1+2^{-\frac{1}{2}+i \tau_{2}}\right)\right)\right|>0.15
$$

for $x_{1}=63, \ldots, 66$, because $x_{2}$ is an integer.

In a last theorem we are summing up the results of this section.

Theorem 7.17. Suppose

1) $17923 \nmid d, a_{2}=2,6.2 \cdot 10^{13} \leq d<10^{574}$, or

2) $17923 \nmid d, a_{2}=3,3 \cdot 10^{13} \leq d<10^{574}$, or

3) $17923 \nmid d, a_{2} \geq 5,2 \cdot 10^{13} \leq d<10^{574}$, or

4) $17923 \mid d, 1.1 \cdot 10^{14} \leq d<10^{574}$.

Then $h(-d) \neq 6$.

\section{BIBLIOGRAPHY}

1. S. Arno, Class number 4, Dissertation, Stanford, 1986.

2. S. Arno, The imaginary quadratic fields of class number 4, Acta Arith. 60 (1992), 321-334. MR 93b: 11144

3. S. Böcherer, Das Gauß'sche Klassenzahlproblem, Mitt. Math. Ges. Hamburg 11 (1988), 565-589. MR 89b:11082

4. D. Goldfeld, The class number of quadratic fields and the conjectures of Birch and SwinnertonDyer, Ann. Scuola Norm. Sup. Pisa (IV) 3 (1976), 623-663. MR 56:8529

5. D. Goldfeld, The conjectures of Birch and Swinnerton-Dyer and the class numbers of quadratic fields, Journées Arithmétiques de Caen 41-42 (1977), 219-227. MR 56:5491

6. D. Goldfeld, Gauss' class number problems for imaginary quadratic fields, Bull. Amer. Math. Soc. 13 (1985), 23-37. MR 86k:11065

7. B. Gross and D. Zagier, Points de Heegner et dérivées de fonctions L, C. R. Acad. Sci. Paris 297 (1983), 85-87. MR 85d:11062

8. B. Gross and D. Zagier, Heegner points and derivatives of L-series, Invent. Math. 84 (1986), 225-230. MR 87j:11057

9. H. Heilbronn, On the class number in imaginary quadratic fields, Quart. J. Math. Oxford Ser. 5 (1934), 150-160.

10. D. H. Lehmer, E. Lehmer and D. Shanks, Integer sequences having prescribed quadratic character, Math. Comp. 24 (1970), 433-451. MR 42:5889

11. H. Montgomery and P. Weinberger, Notes on small class numbers, Acta Arith. 24 (1974), 529-542. MR 50:9841

12. J. Oesterlé, Nombres de classes des corps quadratiques imaginaires, Séminaire Bourbaki 631 (1984), 309-323. MR 86k:11064

13. J. Oesterlé, Le problème de Gauss sur le nombre de classes, Enseign. Math. 34 (1988), 43-67. MR 89j:11108

14. J.-P. Serre, $\triangle=b^{2}-4 a c$, Math. Medley 13 (1985), 1-10. MR 87g:11148

15. D. Shanks, Systematic examination of Littlewood's bounds on $L(1, \chi)$, Proc. Sympos. Pure Math., Analytic Number Theory 24 (1972), 267-269. MR 49:2596 
16. H. Stark, On complex quadratic fields with class-number two, Math. Comp. 29 (1975), 289-302. MR 51:5548

17. Ch. Wagner, Klassenzahl 5, 6 und 7, Dissertation, Freiburg i. Br., 1992.

18. D. Zagier, L-series of elliptic curves, the Birch-Swinnerton-Dyer conjecture, and the class number problems of Gauss, Notices Amer. Math. Soc. 31 (1984), 739-743. MR 86f:11047

Mathematisches Institut, Albertstrasse 23B, 79104 Freiburg i. Br., Germany

Current address: Am Krayenrain 8, 4056 Basel, Switzerland 